\title{
MicroRNAs: Potential Targets in Diabetic Retinopathy
}

Authors

Xin Li, Zi-Wei Yu, Ying Wang, Yu-Hong Fu, Xin-Yuan Gao $\mathbb{D}$
People's Republic of China

Tel.: + 86 18247140616, Fax: 045185555062

15369337970@163.com

\section{ABSTRACT}

Diabetic retinopathy (DR), a serious microvascular complication of diabetes, is a leading cause of blindness in adults. The pathogenesis of DR involves a variety of tissues and complex mechanisms, such as inflammation, oxidative stress, optic neurodegeneration, and autophagy. Nowadays, microRNAs (miRNAs), a novel group of non-coding small RNAs, have been extensively studied and recognized to play a key role in the pathogenesis of DR through aforementioned pathways. Furthermore, some miRNAs have been proposed as biomarkers that may be utilized to screen for DR. Also, miRNAs are a new therapy for DR. In this review, we summarize several miRNAs and, their roles in the pathogenesis of DR. miRNAs, as potential pharmacological targets for the diabetic retinopathy, may provide new insights for the treatment of DR.

\author{
Correspondence \\ Xin-Yuan Gao \\ Department of Endocrinology, The First Affiliated Hospital of \\ Harbin Medical University \\ 150001 Harbin \\ Heilongjiang
}

\section{Introduction}

miRNAs are single-stranded non-coding small RNAs (19-22 nucleotides) generated from a well-organized RNA processing event, and they also belong to highly conserved endogenous RNA sequences. Thus far, approximately 2000 miRNAs across the human genome have been identified and play an important role in physiology and pathophysiology [1]. miRNAs are found to express in all human celltypes and are involved in the major biological processes including cell growth, differentiation, and apoptosis [2]. In the last decade, miRNAs have received substantial attention as potential players involved in microvascular complications of diabetes, affecting the kidney, retina, and peripheral neurons. Compelling evidence indicates that abnormally expressed miRNAs have pivotal roles in key pathogenic processes of microvascular complications [3], such as oxidative stress, apoptosis, inflammation, and angiogenesis. miRNAs have become one of the hot issues after siRNA in recent years.

\section{Circulating miRNAs as Biomakers in DR}

Although miRNAs are not involved in gene coding, they play a regulatory role in Eukaryotic gene expression, cell and individual de- velopment. Upregulation of miRNA can be used to identify functional phenotype, and the inhibition or downregulation can be investigated for functional deletion phenotypes. The combination of upregulation and downregulation can be used to identify the gene regulated by a specific miRNA, as well as cell process affected by the specific miRNA. It is speculated that miRNA regulates one third of the human genes [4]. Meanwhile, some breakthrough study in 2016 [5] demonstrated that miRNAs are also extracellular, being present in a cell-free circulating form in the serum, plasma, saliva, tears, aqueous and vitreous humor, and urine [6,7], which could be called circulating miRNAs.

Zampetaki et al. [8] found that miR-27b and miR-320a are being significantly and independently associated with higher risk of DR. It is an important advance in the field of miRNAs biomarkers that has implications in the areas of DR pathogenesis, prognostication, monitoring, and therapeutics. Compared to tissue-derived miRNAs, circulating miRNAs are more stable. Cells release miRNAs into the circulation, where they have a long-life span (approximately 2 weeks); their stability in plasma/serum/urine on freeze-thawing, efficient recovery, and availability of quantitative detection methods enhance their use as a biomarker as well as a potential media- 
tor of physiological and pathological processes [9]. Therefore, miRNAs are very feasible as novel biomarkers for certain diseases, such as DR.

As biomarkers in DR, miRNAs not only expressed in serum, but also occur in vitreous humor. Notably, in the same individuals, only a few circulating miRNAs were detected in vitreous compared with serum [10]. In > Table 1, we have summarized miR-126 [11, 12], miR-211 [13], miR-93[14-16], miR-122 [17], miR-221 [18], miR27b and miR-320a [8], miR-150-5p, miR-21-3p and miR-30b-5p [19] as biomarkers in DR over the past 5 years. At the same time, we have focused on miRNAs in vitreous humor (VH) of DR, such as miR-126 [20], miR-19a and 27a [21], miR-29a [22, 23], miR-93 and 20a [21], and miR-200b [24] in > Table 2.

\section{miRNAs and Diabetic Retinopathy (DR)}

DR is a progressive disease, which is duration-dependent and develops in stages of growing severity, with oxidative stress, inflammation, and angiogenesis etc. leading to microvascular alterations. Xiong et al. [25] found that miRNAs perform critical regulatory functions during the early stages of DR evolution, including development, differentiation, proliferation and stress responses in DR pathogenesis [26]. Generally, there are two major DR stages non-proliferative (NPDR) and proliferative (PDR) retinopathy. In NPDR, thickening of the basement membrane and pericytes/endothelial cell loss result in increased vascular permeability and development of microvascular abnormalities, such as dilated vessels, capillary microaneurysms, shunts, and vascular occlusion. In PDR, both leaking and breaking of immature and fragile new vessels can cause vitreous hemorrhages, macula edema, fibrosis, retinal detachment, and possibly sight loss [3]. Here, we present a summary and discuss the possibility of targeting miRNAs in potential treatment of DR, providing new ideas for prevention and treatment of DR.

\section{miRNAs, Oxidative Stress, and DR}

Hyperglycemic, a known instigator in DR, could provoke oxidative stress and the studies show that changes in miRNAs expression levels may be associated with the onset and progression of DR. After consulting the literature, we found that miR-7, 15a, 27b, 100, 145, 195, 200b, 365, 383, and miR-455-5p are associated with oxidative stress in DR. Next, we will elaborate on the role of these miRNAs in DR.

\section{miR-7}

Hypoxia is associated with an increment in oxidative stress [27] and the disruption of endothelial adhesion molecules [28, 29], resulting in increased endothelial permeability [30] and impairment of vasodilation [31]. The study of Garcia-Morales et al. [32] showed that hypoxia in DR increases the expression of miR-7, which in turn reduces the protein availability of EPAC-1; the loss of EPAC- 1 in endothelial cells causes endothelial junctional instability and concurrently hyperpermeability, resulting in oxidative stress. Combined, hyperpermeability and oxidative stress might further reduce the oxygen transport creating a feed-forward mechanism that aggravates DR. These results suggest that miR-7 could take part in DR by oxidative stress pathway.
- Table 1 miRNAs as biomarkers in DR and their changes.

\begin{tabular}{|l|l|l|}
\hline miRNAs & Changes in DR & Targets \\
\hline miR-126 & $\uparrow$ & VEGF/IRS-1 \\
\hline miR-211 & $\uparrow$ & Sirtuin1 \\
\hline miR-93 & $\downarrow$ & VEGF \\
\hline miR-122 & $\uparrow / \downarrow$ & - \\
\hline miR-221 & $\uparrow$ & - \\
\hline miR-27b & $\downarrow$ & Antiangiogenic \\
\hline miR-320a & $\uparrow$ & Thrombospondin-1 \\
\hline miR-150-5p & $\downarrow$ & - \\
\hline miR-21-3p & $\uparrow$ & - \\
\hline miR-30b-5p & $\uparrow$ & - \\
\hline
\end{tabular}

- Table 2 miRNAs in vitreous humor (VH) of DR.

\begin{tabular}{|l|l|l|}
\hline miRNAs & $\begin{array}{l}\text { Change } \\
\text { in VH }\end{array}$ & Significance \\
\hline
\end{tabular}

$\operatorname{miR}-126 \quad \uparrow$

miR-126 is closely related to the pathogenesis and progression of PDR, and the miR-126 expression level rises with the increase of severity of PDR

\begin{tabular}{l|l|l} 
miR-19a & $\uparrow$ & miR-19a and miR-27a combined are
\end{tabular} \begin{tabular}{l|l} 
miR-27a & putative diagnostic biomarkers for PDR
\end{tabular}

miR-29a $\quad \downarrow$ miRNA-29a has been shown to be a negative regulator of DR and its decrease in expression levels is associated with exacerbation of PDR

$\operatorname{miR}-93 \quad \uparrow$

miR-20a

miRNA-20a and miRNA-93 could suggest a prognostic biomarker potential for sight-threatening PDR complications such as recurrent vitreous hemorrhage (RVH)

miR-200b $\quad \uparrow$ Both miRNA-200b and VEGF are increased in the vitreous of patients with PDR but in a noncorrelated pattern. miRNA-200b may be involved in the pathogenesis of PDR but through VEGF-independent mechanisms

Further studies are needed to identify the miRNA-200b targeted genes that are involved in PDR pathogenesis and to examine the potential role of miRNA-200b as a target for PDR treatment

miR-15a, miR-27b, miR-145, miR-383

Many studies have shown that reactive oxygen species (ROS) is a major causative factor involved in the development of DR [33]. Based on the study of Kamalden et al. [34], miR-15a, produced in pancreatic $\beta$-cells, can enter the bloodstream and contribute to retinal injury. They found that miR-15a overexpression results in oxidative stress by targeting Akt3 and by inhibiting PI-3 kinase path- 
way to induce ROS accumulation, which lead to apoptotic cell death. In other words, miR-15a is correlated with diabetes severity and may contribute to the development of DR.

Li, Hui, Kang et al. [35] found that miR-27b reduces the generation of ROS and downregulates the P13K/AKT/mTOR signaling pathway by inhibition of Nox2. Hui and Yin [36] found miR-145 overexpression reduced the intracellular ROS production and malondialdehyde level, whereas it increased the activity of superoxide dismutase. Therefore, miR-145 can reduce high-glucose induced oxidative stress and apoptosis in retinal endothelial cells. In addition, PRDX3 is a target of miRNA-383, which can play a role in cell survival by decreasing the ROS [37, 38]. Meanwhile, miR-383 mediates high glucose-induced oxidative stress and apoptosis in retinal pigment epithelial (RPE) cells by repressing peroxiredoxin 3 (PRDX3) [39]. These miRNAs may have an impact on the treatment or prevention of DR.

\section{miR-100}

miR-100, a member of miR-99 family (including miR-99a, miR-99b, miR-100), is a key apoptotic regulator in various cell types [40, 41]. Recently, it has been discovered that oxidative stress can upregulate miR-100 in retinal ganglion cells (RGCs), and hydrogen peroxide can induce apoptosis by increasing miR-100 expression. Conversely, diminished miR-100 expression is associated with activation of the AKT pathway, extracellular-signal-regulated kinase (ERK) pathway, and tropomyosin receptor kinase B (TrkB) pathway [42]. And a study in 2014 [43] has found IGF1R (IGF-1 receptor) was directly regulated by miR-100 in RGC-5 cells, and siRNA-mediated IGF1R knockdown activated AKT protein through phosphorylation; downregulated miR-100, thus exerted a protective effect on RGC-5 apoptosis. Therefore, reducing miR-100 expression can be an applied therapeutic method to protect oxidative stress-induced apoptosis.

\section{miR-195}

There is an established association between miR-195 and oxidative stress/diabetes-induced retinal endothelial cell injury because of miR-195 acting as a regulator for mitofusin-2 (MFN2), which is known to be involved in oxidative stress and diabetes associated complications [42]. Based on the luciferase report assay, miR-195 binds to the 3'-UTR of MFN2 mRNA and its overexpression can result in the downexpression of MFN2 protein leading to tube formation and an increase in blood-retinal barrier permeability [44], thus leads to DR. Therefore, miR-195 could be a potential therapeutic target for intervention of DR in future [45].

\section{miR-200b}

miR-200b is one of the well-known miRNAs, which could affect DR by affecting oxidative stress. Although bioinformatic analyses identified a number of potential target genes of miR-200b, we focus on oxidation resistance 1 (Oxr1) as a downstream target gene regulated by miR-200b. A study represented that miR-200 can downregulate Oxr1 expression in the retina of diabetic rats as increasing the expression of Oxr1 induces oxidative stress and apoptosis [46]. These results suggested that miR-200b-regulated Oxr1 potentially has a protective role in DR.
miR-365 and miR-455-5p

In the retina, miR-365 was mainly expressed in the INL (inner nuclear layer), PR layer, and RPE (retinal pigment epithelial) layer, especially in Müller cells. Based on a study by Wang et al.[47], Timp3 is a target of miR-365 and is negatively regulated by miR-365. They demonstrated that the increase of miR-365 in retinal Müller cells participated in the pathogenesis of DR through oxidative stress, and miR-365 inhibits Timp3 to increase oxidative stress. In other words, miR-365/Timp3 could be a potential therapeutic target for treating DR.

Suppressor of cytokine signaling 3 (SOCS3) is a direct target gene of miR-455-5p and negatively regulated by miR-455-5p. The study showed that augmentation of miR-455-5p remarkably alleviated HG-triggered oxidative stress injury as reflected by decreased the production of intracellular ROS and malondialdehyde (MDA) content as well as NADPH oxidase 4 expression, concomitant with enhanced the activities of superoxide dismutase, catalase, and GPX [48]. That is to say, miR-455-5p has antioxidant activity and could be as a new potential therapeutic agent for DR treatment.

\section{miRNAs, Inflammation, and DR}

Hyperglycemia stimulates inflammation and promotes vascular dysfunction of the retina, leading to increased capillary permeability and vascular leakage. Therefore, inflammation signaling pathways play a major role in DR. miRNAs can interact with mRNA on miRNA response element that is found in the UTR of the mRNA target. With that, miRNAs regulate almost every cellular and developmental process, including the regulation of instinct immune responses and inflammation [49]. Herein we could summarize the role of miRNAs in inflammation pathways of DR. After consulting the literature, we found that miR-15a, 145, 146 are associated with inflammation in DR.

miR-15a, miR-15a/16, and miR-145

miR-15a is a key regulator of both pro-inflammatory and pro-angiogenic pathways and is downregulated in diabetic retina. Based on a study conducted by Wang et al. [50], miR-15a was identified as a miRNA that provides inhibition to both ASM and VEGF-A activation. They previously found that acid sphingomyelinase (ASM), the enzyme converting sphingomyelin into pro-inflammatory and pro-apoptotic ceramide, is highly activated by diabetes in the retina [51]. Endothelial cells, which represent a major source of ASM, had the highest level of activation of ASM in diabetic retina. Therefore, they provide an entirely new mechanism for the pathogenesis of DR based on diabetes-induced downregulation of miR-15a expression leading to pro-inflammatory and pro-angiogenic changes in the diabetic retina due to unopposed activation of miR-15a target genes, ASM and VEGF-A [50].

In addition, the evidence showed that hyperglycemia can induce downregulation of miR-15a and miR-16, while overexpression of miR-15a and miR-16 can decrease pro-inflammatory signaling pathways of IL-1 $\beta$, TNF- $\alpha$, and NF-KB under conditions of hyperglycemia. Also, decrease of miR-15a and miR-16 induce retinal leukocytosis and increase CD45 levels, followed by upregulated levels of IL-1 $\beta$, TNF- $\alpha$, and NF-KB pathways [52]. Consequently, miR-15a 
and miR-16 can play essential roles in reducing retinal leukocytosis, probably through inhibition of inflammatory cellular signaling pathways [53].

Based on a study by Hui and Yin [36], miR-145 attenuates high glucose-induced oxidative stress and inflammation in retinal endothelial cells through regulating TLR4/NF-KB signaling. However, other mechanisms by which miR-145 affects the DR inflammatory pathway remain to be investigated.

\section{miR-146}

miR-146a is a well-known modulator of both the innate and adaptive immune response, which has been implicated to function in inflammation, innate immunity, and cancer. miR-146a has also been shown to regulate mitochondrial functions such as inflammation-aging [54-56]. A previous study showed miR-146a expression in different types of retinal cells, including retinal endothelial cells, Müller cells, and RPE cells [57]. One of the most important miRNAs in retinal endothelial cells is miR-146a, which is transactivated by NF-KB pathways for the upregulation of this expression. The increase of miR-146a expression levels also exerts negative feedback on IL-1R/TLR-mediated NF-kB activation by targeting IL- 1 receptor-associated kinase 1 (IRAK1) and TNF- $\alpha$ receptor-associated factor-6. Thus, miR-146a can play a negative regulatory function in NF-kB activation pathways, which are related to inflammatory processes in DR [58].

In addition, a previous study showed an increase of ADA2 (AdenosineDeaminase-2) in macrophage-rich tissues during inflammatory $[59,60]$ and ADA2 activity is elevated significantly in pleural fluids of patients from patients with diabetes [61]. Then, it was found that ADA2 is a direct target of miR-146b-3p and decreased miR-146b-3p is associated with increased ADA2 activity. Ectopic expression of miR-146b-3p suppressed ADA2 expression, activity, and TNF-release in the AGA-treated human macrophages. These results suggest a regulatory role of miR-146b-3p in diabetes related retinal inflammation by suppressing ADA2 [62].

Finally, miR-146 may be an alternative therapeutic target for the treatment of diabetic retinopathy through inhibition of NF-KB signaling pathways. [63]. All in all, it is evident that miR-146 plays an important role in the inflammatory pathways that occur in DR. Thus, there is more work to be done in the future.

\section{miRNAs, Angiogenesis, and DR}

Angiogenesis is the process through which new blood vessels arise from the pre-existing post-capillary venule, which is usually the end result of DR, especially as a maker for distinguishing NPDR and PDR. A breakthrough experiment in 2006 first revealed the involvement of miRNAs in angiogenesis [64]. Regulation of angiogenesis is very complex and, the addition of latest dimension of miRNA-based regulation, have made it more complicated [65]. The term angio-miRNAs (angio-miRs) were proposed to miRNAs that regulate angiogenesis [66]. As we all know, a wide range of regulators and signaling molecules, including vascular endothelial growth factor-A (VEGF-A), fibroblast growth factor (FGF), epidermal growth factor (EGF), interferon, matrix metalloproteinase-1/9 (MMP-1/9) are associated with angiogenesis [67-69]. Herein, we have summarized some miRNAs that have impacts on DR by VEGF and FGF.
miRNAs can be divided in two types, one is pro-angiogenic and the other is anti-angiogenic. miR-126, miR-17-92 cluster, let-7b, 7f, miR-130, miR210, miR-378, and miR-296 are reported as pro-angiomiRs and miR-221/222, miR-328, miR-15b/miR-16, and miR-20a/20b function as anti-angiomiRs [70].

miR-126 is an endothelial specific miRNA, which plays a pivotal role in maintaining endothelial homeostasis and vascular integrity [71]. In response to VEGF and bFGF, it suppresses the negative regulators of angiogenesis such as sprout-related protein (SPRED-1) and $\mathrm{PI} 3$ kinase regulatory subunit 2 (PIK3R2), and promotes angiogenesis [11]. miR-200b is a cluster of miR-200 family, which are highly associated with epithelial-mesenchymal transition (EMT). VEGF-a gene is a direct target gene of miR-200b and its overexpression can reduce the growth level of retinal micro-vessel density (MVD) so as to reverse the occurrence of retinal lesions and can also reduce the number of vascular endothelial cells and inhibit the formation of retinal neovascularization buds [72]. Recently, miR-15a has been identified as a key regulator of pro-angiogenic pathway through direct binding to VEGF-a (but not VEGF-b, $c$ and d) 3'UTR [73]. Based on the study of Wang et al. [50], miR-15a negatively regulates the expression level of VEGF-a. And VEGF-a and FGF2, by miR-15a is important in maintaining the delicate balance. Disruption of this balance could favor pathological angiogenesis, such as seen in DR. In addition, miR-15b and miR-16 have VEGF-a as their predicted target, which they are down regulated with hypoxia induction and promote VEGF-a expression which ultimately facilitates angiogenesis [74].

Finally, we introduce one of the family of miR-21, miR-21-5p. miR-21 is highly expressed in a variety of cancers [75-77] and diabetes-related diseases [78]. However, the effect of miR-21 on retinal angiogenesis is still not clear. In the present study, human retinal microvascular endothelial cells (HRMECs) were employed to investigate the effect of miR-21-5p on high glucose-induced angiogenesis and the underlying mechanisms. They found inhibition of miR-21-5p suppresses high glucose-induced proliferation and angiogenesis of human retinal microvascular endothelial cells by the regulation of AKT and ERK pathways via maspin [79], which is a target gene of miR-21-5p that involved in angiogenesis [80]. In conclusion, there are a great deal of miRNAs associated with angiogenesis, but angio-miRNAs in DR are still studied.

\section{miRNAs and Therapy}

Nowadays, targeting miRNAs is becoming a novel therapeutic approach. Developing miRNAs-based therapeutics has two major approaches: antagonists to inhibit endogenously present miRNA that has lethal gain of function and miRNA mimics, which can restore useful miRNAs, with the loss of function [81]. miRNA mimics are small, chemically modified, double stranded RNA molecules that can mimic endogenous mature miRNA molecules [82]. Both miRNA mimics and inhibitors have been delivered at the target tissues by use of numerous conjugate molecules in the experimental setting [83].

A study by Zhang, Cui, and Xu [84] found that FGF5 is a target gene of miR-145-5p and FGF5 knockdown could partially reverse the protective effects of miR-145-5p on RGC-5 cells. And miR-145$5 p$ inhibitor decreased pro-inflammatory cytokines, including 
tumor necrosis factor- $\alpha$ (TNF- $\alpha$ ), interleukin- 6 (IL-6) levels, elevated cell viability and proliferation, as well as suppressed cell apoptosis. In other words, miR-145-5p inhibitor might be a neuroprotective target for DR. A new study found that miR-183 inhibitor suppressed the proliferation and angiogenesis of vascular endothelial cells by inactivating the PI3K/Akt/VEGF signaling pathway via the downregulation of BTG1, which may be a new therapeutic target for DR [85].

In addition, the study of Chen, Zhao, and Gu et al. [86] found FGF16 is a target gene of miR-144-3p and miR-144-3p inhibited high glucose-induced cell proliferation through suppressing FGF16 and MAPK signaling pathway, suggesting it may be novel targets for DR prevention. Also the current study supports that miR-384$3 p$ inhibits the proliferation of RMECs through downregulating HK2 (hexokinase 2) and provides inhibition of retinal neovascularization in $\mathrm{DR}$, which provides significant guidance for further exploring the therapeutic methods for DR [87].

In the future, miRNA mimics and inhibitors may be an effective way to treat DR, and studying the mechanism of miRNAs in DR may provide a new idea for DR therapy.

\section{Conclusion and Perspective}

In conclusion, miRNAs are involved in the pathogenesis of DR in various ways, including inflammation, oxidative stress, and angiogenesis. Studying their roles in DR could lead to a more detailed understanding of the development of the disease and its effective treatment. With the discovery of more miRNAs, miRNAs as biomarkers in DR has become a research hotspot. In addition to miR27b and miR-320a, miR-150-5p and others have been found to act as biomarkers. Changes in miRNAs levels can be detected in diabetics at present, however, in the future we may find changes in miRNAs levels could distinguish the stages of DR or the type of diabetes. It is promising to use miRNAs to treat DR, but the specific pathophysiological processes of miRNAs and their target genes in DR still need to be fully studied and verified. miRNA mimics or inhibitors are now widely used in animal studies and may one day be a new way to treat DR. In order to reduce the risk of blindness in diabetic patients, we should further study the target of miRNAs and their effect on DR.

\section{Funding Information}

This work was supported by a grant from the Natural Foundation of Heilongjiang Province (grant number H2015057, China).

\section{Conflict of Interest}

The authors declare that they have no conflict of interest.

\section{References}

[1] Yu J, Su W, Zhang X et al. MicroRNAs in type 2 diabetes mellitus: Important for the pathogenesis but uncertain as biomarkers. J Diabetes 2018; DOI:10.1111/1753-0407.12772
[2] Bartel DP. MicroRNAs: Genomics, biogenesis, mechanism, and function. Cell 2004; 116: 281-297

[3] Barutta F, Bellini S, Mastrocola R et al. MicroRNA and microvascular complications of diabetes. Int J Endocrinol 2018; 6890501: 1-20

[4] Armand-Labit V, Pradines A. Circulating cell-free microRNAs as clinical cancer biomarkers. Biomol Concepts 2017; 8: 61-81

[5] Fehlmann T, Ludwig N, Backes $C$ et al. Distribution of microRNA biomarker candidates in solid tissues and body fluids. RNA Biol 2016; 13: $1084-1088$

[6] Ye EA, Steinle J]. miR-146a suppresses STAT3/VEGF pathways and reduces apoptosis through IL-6 signaling in primary human retinal microvascular endothelial cells in high glucose conditions. Vision Res 2017; 139: 15-22

[7] Shaker OG, Abdelaleem OO, Mahmoud RH et al. Diagnostic and prognostic role of serum miR-20b, miR-17-3p, HOTAIR, and MALAT1 in diabetic retinopathy. IUBMB Life 2019; 71: 310-320

[8] Zampetaki A, Willeit P, Burr S et al. Angiogenic microRNAs linked to incidence and progression of diabetic retinopathy in type 1 diabetes. Diabetes 2016; 65: 216-227

[9] Joglekar MV, Januszewski AS, Jenkins AJ et al. Circulating microRNA biomarkers of diabetic retinopathy. Diabetes 2016; 65: 22-24

[10] Ragusa M, Caltabiano R, Russo A et al. MicroRNAs in vitreus humor from patients with ocular diseases. Mol Vis 2013; 19: 430-440

[11] Qin LL, An MX, Liu YL et al. MicroRNA-126: A promising novel biomarker in peripheral blood for diabetic retinopathy. Int J Ophthalmol 2017; 10: 530-534

[12] Fang S, Ma X, Guo S et al. MicroRNA-126 inhibits cell viability and invasion in a diabetic retinopathy model via targeting IRS-1. Oncol Lett 2017; 14: 4311-4318

[13] Liu HN, Cao NJ, Li X et al. Serum microRNA-211 as a biomarker for diabetic retinopathy via modulating Sirtuin 1 . Biochem Biophys Res Commun 2018; 505: 1236-1243

[14] Zou HL, Wang Y, Gang Q et al. Plasma level of miR-93 is associated with higher risk to develop type 2 diabetic retinopathy. Graefes Arch Clin Exp Ophthalmol 2017; 255: 1159-1166

[15] Hirota $\mathrm{K}$, Keino $\mathrm{H}$, Inoue $\mathrm{M}$ et al. Comparisons of microRNA expression profiles in vitreous humor between eyes with macular hole and eyes with proliferative diabetic retinopathy. Graefes Arch Clin Exp Ophthalmol 2015; 253: 335-342

[16] Salam A, Mathew R, Sivaprasad S. Treatment of proliferative diabetic retinopathy with anti-VEGF agents. Acta Ophthalmol 2011; 89: 405-411

[17] Pastukh N, Meerson A, Kalish D et al. Serum miR-122 levels correlate with diabetic retinopathy. Clin Exp Med 2019; 19: 255-260

[18] Liu HN, Li X, Wu N et al. Serum microRNA-221 as a biomarker for diabetic retinopathy in patients associated with type 2 diabetes. Int J Ophthalmol 2018; 11: 1889-1894

[19] Mazzeo A, Beltramo E, Lopatina T et al. Molecular and functional characterization of circulating extracellular vesicles from diabetic patients with and without retinopathy and healthy subjects. Exp Eye Res 2018; 176: 69-77

[20] Liu R, Liu CM, Cui LL et al. Expression and significance of MiR-126 and VEGF in proliferative diabetic retinopathy. Eur Rev Med Pharmacol Sci 2019; 23: 6387-6393

[21] Mammadzada P, Bayle J, Gudmundsson J et al. Identification of diagnostic and prognostic micrornas for recurrent vitreous hemorrhage in patients with proliferative diabetic retinopathy. J Clin Med. 2019; 8: 1-14

[22] Gong Q, Su G. Roles of miRNAs and long noncoding RNAs in the progression of diabetic retinopathy. Biosci Rep 2017; 37: 1-18. DOI:10.1042/BSR20171157 
[23] Zhang J, Wu L, Chen J et al. Downregulation of MicroRNA 29a/b exacerbated diabetic retinopathy by impairing the function of Muller cells via Forkhead box protein O4. Diab Vasc Dis Res 2018; 15 : 214-222

[24] Gomaa AR, Elsayed ET, Moftah RF. MicroRNA-200b Expression in the vitreous humor of patients with proliferative diabetic retinopathy. Ophthalmic Res 2017; 58: 168-175

[25] Xiong F, Du X, Hu J et al. Altered retinal microRNA expression profiles in early diabetic retinopathy: An in silico analysis. Curr Eye Res 2014; 39: $720-729$

[26] Natarajan R, Putta S, Kato M. MicroRNAs and diabetic complications. J Cardiovasc Transl Res 2012; 5: 413-422

[27] Pearlstein DP, Ali MH, Mungai PT et al. Role of mitochondrial oxidant generation in endothelial cell responses to hypoxia. Arterioscler Thromb Vasc Biol 2002; 22: 566-573

[28] Yan SF, Ogawa S, Stern DM et al. Hypoxia-induced modulation of endothelial cell properties: regulation of barrier function and expression of interleukin-6. Kidney Int 1997; 51: 419-425

[29] Koto T, Takubo K, Ishida $S$ et al. Hypoxia disrupts the barrier function of neural blood vessels through changes in the expression of claudin-5 in endothelial cells. Am J Pathol 2007; 170: 1389-1397

[30] Ten VS, Pinsky D]. Endothelial response to hypoxia: physiologic adaptation and pathologic dysfunction. Curr Opin Crit Care 2002; 8: 242-250

[31] Han JA, Seo EY, Kim H] et al. Hypoxia-augmented constriction of deep femoral artery mediated by inhibition of eNOS in smooth muscle. Am J Physiol Cell Physiol 2013; 304: C78-C88

[32] Garcia-Morales V, Friedrich J, Jorna LM et al. The microRNA-7-mediated reduction in EPAC-1 contributes to vascular endothelial permeability and eNOS uncoupling in murine experimental retinopathy. Acta Diabetol 2017; 54: 581-591

[33] Volpe CMO, Villar-Delfino PH, Dos Anjos PMF et al. Cellular death, reactive oxygen species (ROS) and diabetic complications. Cell Death Dis 2018; 9: 119

[34] Kamalden TA, Macgregor-Das AM, Kannan SM et al. Exosoma microRNA-15a transfer from the pancreas augments diabetic complications by inducing oxidative stress. Antioxid Redox Signal 2017; 27: 913-930

[35] Li ], Hui L, Kang Q et al. Down-regulation of microRNA-27b promotes retinal pigment epithelial cell proliferation and migration by targeting Nox2. Pathol Res Pract 2018; 214: 925-933

[36] Nguyen TA, Park J, Dang TL et al. Microprocessor depends on hemin to recognize the apical loop of primary microRNA. Nucleic Acids Res 2018; 46: 5726-5736

[37] Li KK, Pang JC, Lau KM et al. MiR-383 is downregulated in medulloblastoma and targets peroxiredoxin 3 (PRDX3). Brain Pathol 2013; 23: 413-425

[38] Wang YG, Li L, Liu CH et al. Peroxiredoxin 3 is resistant to oxidation-induced apoptosis of Hep-3b cells. Clin Transl Oncol 2014; 16: 561-566

[39] Jiang Y, Sang Y, Qiu Q. microRNA-383 mediates high glucose-induced oxidative stress and apoptosis in retinal pigment epithelial cells by repressing peroxiredoxin 3. Am J Transl Res 2017; 9: 2374-2383

[40] Nagaraja AK, Creighton CJ, Yu Z et al. A link between mir-100 and FRAP1/mTOR in clear cell ovarian cancer. Mol Endocrinol 2010; 24: 447-463

[41] Shi W, Alajez NM, Bastianutto C et al. Significance of Plk1 regulation by miR-100 in human nasopharyngeal cancer. Int J Cancer 2010; 126: 2036-2048

[42] Satari M, Aghadavod E, Mobini M et al. Association between miRNAs expression and signaling pathways of oxidative stress in diabetic retinopathy. J Cell Physiol 2019; 234: 8522-8532
[43] Kong N, Lu X, Li B. Downregulation of microRNA-100 protects apoptosis and promotes neuronal growth in retinal ganglion cells. BMC Mol Biol 2014; 15: 25

[44] Zhang R, Garrett Q, Zhou H et al. Upregulation of miR-195 accelerates oxidative stress-induced retinal endothelial cell injury by targeting mitofusin 2 in diabetic rats. Mol Cell Endocrinol 2017; 452: 33-43

[45] Zhong Q, Kowluru RA. Diabetic retinopathy and damage to mitochondrial structure and transport machinery. Invest Ophthalmol Vis Sci 2011; 52: 8739-8746

[46] Murray AR, Chen Q, Takahashi Y et al. MicroRNA-200b downregulates oxidation resistance $1(\mathrm{Oxr} 1)$ expression in the retina of type 1 diabetes model. Invest Ophthalmol Vis Sci 2013; 54: 1689-1697

[47] Wang J, Zhang J, Chen X et al. miR-365 promotes diabetic retinopathy through inhibiting Timp3 and increasing oxidative stress. Exp Eye Res 2018; 168: 89-99

[48] Chen P, Miao Y, Yan P et al. MiR-455-5p ameliorates HG-induced apoptosis, oxidative stress and inflammatory via targeting SOCS3 in retinal pigment epithelial cells. J Cell Physiol 2019; 234: 21915-21924

[49] Nejad C, Stunden H], Gantier MP. A guide to miRNAs in inflammation and innate immune responses. FEBS J 2018; 285: 3695-3716

[50] Wang Q, Navitskaya S, Chakravarthy H et al. Dual anti-inflammatory and anti-angiogenic action of miR-15a in diabetic retinopathy. EBioMedicine 2016; 11: 138-150

[51] Opreanu M, Tikhonenko M, Bozack S et al. The unconventional role of acid sphingomyelinase in regulation of retinal microangiopathy in diabetic human and animal models. Diabetes 2011; 60: 2370-2378

[52] Shafabakhsh R, Aghadavod E, Mobini M et al. Association between microRNAs expression and signaling pathways of inflammatory markers in diabetic retinopathy. J Cell Physiol 2019; 234: 7781-7787

[53] Ye EA, Liu L, Jiang Y et al. miR-15a/16 reduces retinal leukostasis through decreased pro-inflammatory signaling. | Neuroinflammation 2016; 13: 305

[54] Li Y, VandenBoom TG 2nd, Wang Z et al. Up-regulation of miR-146a contributes to the inhibition of invasion of pancreatic cancer cells. Cancer Res 2010; 70: 5703

[55] Nahid MA, Satoh M, Chan EK. Mechanistic role of microRNA-146a in endotoxin-induced differential cross-regulation of TLR signaling. J Immunol 2011; 186: 1723-1734

[56] Rippo MR, Olivieri F, Monsurro $V$ et al. MitomiRs in human inflamm-aging: a hypothesis involving miR-181a, miR-34a and miR-146a. Exp Gerontol 2014; 56: 154-163

[57] Wang Q, Bozack SN, Yan Y et al. Regulation of retinal inflammation by rhythmic expression of MiR-146a in diabetic retina. Invest Ophthalmol Vis Sci 2014; 55: 3986-3994

[58] Ye EA, Steinle J]. miR-146a attenuates inflammatory pathways mediated by TLR4/NF-kappaB and TNFalpha to protect primary human retinal microvascular endothelial cells grown in high glucose. Mediators Inflamm 2016; 2016: 3958453

[59] Gorguner M, Cerci M, Gorguner I. Determination of adenosine deaminase activity and its isoenzymes for diagnosis of pleural effusions. Respirology 2000; 5: 321-324

[60] Ungerer JP, Oosthuizen HM, Retief JH et al. Significance of adenosine deaminase activity and its isoenzymes in tuberculous effusions. Chest 1994; 106: 33-37

[61] Hoshino T, Yamada K, Masuoka K et al. Elevated adenosine deaminase activity in the serum of patients with diabetes mellitus. Diabetes Res Clin Pract 1994; 25: 97-102

[62] Fulzele S, El-Sherbini A, Ahmad S et al. MicroRNA-146b-3p regulates retinal inflammation by suppressing adenosine deaminase- 2 in diabetes. Biomed Res Int. 2015; 846501: 1-8 
[63] Zhuang P, Muraleedharan CK, Xu S. Intraocular delivery of miR-146 inhibits diabetes-induced retinal functional defects in diabetic rat model. Invest Ophthalmol Vis Sci 2017; 58: 1646-1655

[64] Poliseno L, Tuccoli A, Mariani L et al. MicroRNAs modulate the angiogenic properties of HUVECs. Blood 2006; 108: 3068-3071

[65] Kuehbacher A, Urbich C, Zeiher AM et al. Role of Dicer and Drosha for endothelial microRNA expression and angiogenesis. Circ Res 2007; 101: 59-68

[66] Chang SH, Hla T. Gene regulation by RNA binding proteins and microRNAs in angiogenesis. Trends Mol Med 2011; 17: 650-658

[67] Ribatti D, Conconi MT, Nussdorfer GG. Nonclassic endogenous novel [corrected] regulators of angiogenesis. Pharmacol Rev 2007; 59: 185-205

[68] Chung AS, Lee J, Ferrara N. Targeting the tumour vasculature: Insights from physiological angiogenesis. Nat Rev Cancer 2010; 10: 505-514

[69] Carmeliet P, Jain RK. Molecular mechanisms and clinical applications of angiogenesis. Nature 2011; 473: 298-307

[70] Wu F, Yang Z, Li G. Role of specific microRNAs for endothelial function and angiogenesis. Biochem Biophys Res Commun 2009; 386: 549-553

[71] van Solingen C, Bijkerk R, de Boer HC et al. The role of microRNA-126 in vascular homeostasis. Curr Vasc Pharmacol 2015; 13: 341-351

[72] Li EH, Huang QZ, Li GC et al. Effects of miRNA-200b on the development of diabetic retinopathy by targeting VEGFA gene. Biosci Rep 2017; 37: 1-25. DOI:10.1042/BSR20160572

[73] Yin KJ, Olsen K, Hamblin M et al. Vascular endothelial cell-specific microRNA-15a inhibits angiogenesis in hindlimb ischemia. J Biol Chem 2012; 287: 27055-27064

[74] Hua Z, Lv Q, Ye W et al. MiRNA-directed regulation of VEGF and other angiogenic factors under hypoxia. PLoS One 2006; 1: e116

[75] Kao HW, Pan CY, Lai CH et al. Urine miR-21-5p as a potential non-invasive biomarker for gastric cancer. Oncotarget 2017; 8: 56389-56397

[76] Qu K, Zhang X, Lin T et al. Circulating miRNA-21-5p as a diagnostic biomarker for pancreatic cancer: evidence from comprehensive miRNA expression profiling analysis and clinical validation. Sci Rep 2017; 7: 1692
[77] Matsuzaki K, Fujita K, Jingushi K et al. MiR-21-5p in urinary extracellular vesicles is a novel biomarker of urothelial carcinoma. Oncotarget 2017; 8: 24668-24678

[78] jiang Q, Lyu XM, Yuan Y et al. Plasma miR-21 expression: An indicator for the severity of Type 2 diabetes with diabetic retinopathy. Biosci Rep 2017; 37: 1-4. DOI:10.1042/BSR20160589

[79] Qiu F, Tong H, Wang Y et al. Inhibition of miR-21-5p suppresses high glucose-induced proliferation and angiogenesis of human retinal microvascular endothelial cells by the regulation of AKT and ERK pathways via maspin. Biosci Biotechnol Biochem 2018; 82: 1366-1376

[80] Chen B, Chen X, Wu X et al. Disruption of microRNA-21 by TALEN leads to diminished cell transformation and increased expression of cell-environment interaction genes. Cancer Lett 2015; 356: 506-516

[81] Caporali A, Emanueli C. MicroRNA regulation in angiogenesis. Vascul Pharmacol 2011; 55: 79-86

[82] van Rooij E, Marshall WS, Olson EN. Toward microRNA-based therapeutics for heart disease: The sense in antisense. Circ Res 2008; 103: 919-928

[83] Wang J, Chen J, Sen S. MicroRNA as biomarkers and diagnostics. J Cell Physiol 2016; 231: 25-30

[84] Zhang J, Cui C, Xu H. Downregulation of miR-145-5p elevates retinal ganglion cell survival to delay diabetic retinopathy progress by targeting FGF5. Biosci Biotechnol Biochem 2019; 83: 1655-1662

[85] Zhang ZZ, Qin XH, Zhang J. MicroRNA-183 inhibition exerts suppressive effects on diabetic retinopathy by inactivating BTG1-mediated PI3K/Akt/VEGF signaling pathway. Am J Physiol Endocrinol Metab 2019; 316: E1050-E1060

[86] Chen C, Zhao C, Gu C et al. MiRNA-144-3p inhibits high glucose induced cell proliferation through suppressing FGF16. Biosci Rep 2019; 39: 6-6. DOI:10.1042/BSR20181788

[87] Xia F, Sun J], jiang YQ et al. MicroRNA-384-3p inhibits retinal neovascularization through targeting hexokinase 2 in mice with diabetic retinopathy. J Cell Physiol 2018; 234: 721-730 